\title{
A Model for Ferroelectric Phase Shifters
}

\author{
R. R. Romanofsky ${ }^{1}$, Senior Member IEEE, and A. H. Qureshi
}

\begin{abstract}
Novel microwave phase shifters consisting of coupled microstriplines on thin ferroelectric films have been demonstrated recently. A theoretical model useful for predicting the propagation characteristics (insertion phase shift, dielectric loss, impedance, and bandwidth) is presented here. The model is based on a variational solution for line capacitance and coupled strip transmission line theory.
\end{abstract}

\section{Index Terms-Ferroelectricity, Phase shifter circuits}

\section{INTRODUCTION}

It is well known that the permittivity of ferroelectric material can be modified by application of a dc electric field. Recently, microwave phase shifters have been demonstrated that used thin ferroelectric films and superconducting or normal metal coupled microstrip lines that also served as biasing electrodes [1-3]. The insertion loss of these Ku- and $\mathrm{K}$-band phase shifters was substantially better than their semiconductor microwave integrated circuit counterparts. More than $400^{\circ}$ of continuous phase shift with about $4 \mathrm{~dB}$ loss at $77 \mathrm{~K}$ was obtained using $\mathrm{YBa}_{2} \mathrm{Cu}_{3} \mathrm{O}_{7 . \delta}$ electrodes and the incipient ferroelectric $\mathrm{SrTiO} 3$, sequentially deposited onto $0.25 \mathrm{~mm}$ thick $\mathrm{LaAlO}_{3}$ substrates. An insertion loss of about $5 \mathrm{~dB}$ was obtained from a similar design using gold electrodes and $\mathrm{Ba}_{\mathrm{x}} \mathrm{Sr}_{1-\mathrm{x}} \mathrm{TiO}_{3}$ films on $\mathrm{LaAlO}_{3}$ at room temperature. The films were grown by pulsed laser ablation. An intriguing possibility is the combination of ferroelectrics and ferromagnetics to allow control of both capacitance and inductance for tuning while maintaining nearly constant impedance $[4,5]$.

These simple phase shifters enable a new type of phased array antenna called a ferroelectric reflectarray [6,7]. A reflectarray antenna combines the best features of a gimbaled parabolic dish (i.e., high efficiency and low cost) and a direct radiating array (e.g., vibration-free scanning). It consists of a surface of printed elements illuminated by a radiating feed. The energy from the feed can be re-radiated to form a cophasal beam. In the past, reflectarray antennas have been implemented using spiral elements interconnected with diodes to achieve far field phase shift [8]. However, their performance has been limited by the losses of the phase shifting elements and the finite number of phase shifting bits. Fixed beam printed microstrip patch reflectarrays have also been reported [9]. But a viable technique for including variable phase shift with printed radiators was elusive. The

\footnotetext{
'Manuscript received May 9, 2000.

R. R. Romanofsky, is with the National Aeronautics and Space Administration, Glenn Research Center, Cleveland, Ohio 44135 (telephone: 216-433-3507, e-mail: Robert.R.Romanofsky@grc.nasa.gov).

A. H. Qureshi, is with the Cleveland State University, Cleveland, Ohio (telephone: 440-892-7936, e-mail: qureshi@csvx.csuohio.edu).
}

phase shifters analyzed here hold promise for this application because they are compact, low-loss, and can be fabricated lithographically on the same surface as the radiating element. Note that the devices are generally operated in the paraelectric regime to minimize loss and hysteresis.

\section{Variational Formulation of Line CapactTance}

The designs are based on a series of coupled microstriplines interconnected with short sections of nominally $50 \Omega$ microstrip. Bias up to $400 \mathrm{~V}$ is applied to the sections via printed bias-tees consisting of a quarter-wave radial stub in series with a very high impedance quarter-wave microstrip. A sketch of the cross-section is shown in Fig. 1. By concentrating the fields in the odd mode, the phase shift per unit length is maximized and by using the film in thin film form the effects of high loss tangent are minimized. The amount of phase shift can be increased by cascading coupled line sections. A figure of merit approaching $120 \% \mathrm{~dB}\left(80^{\circ} / \mathrm{dB}\right)$ has been achieved from cryogenically cooled (room temperature) devices [1-3]. Though methods for calculating the propagation parameters of coupled transmission lines are well known, coupled lines on stratified substrates are difficult to analyze. And the high permittivity of the ferroelectric layer causes unacceptably long computation time by full-wave electromagnetic simulators because the geometry must be fractured into many thousands of cells. The multi-layer structure is analyzed here using a known computationally efficient variational method to calculate the complex propagation constant and characteristic impedance. The method is quite general and can be used for multiple layers of various dielectrics or other types of transmission lines. For example, a multi-layer microstrip can be analyzed by allowing the strip spacing (s) to become much greater than the substrate thickness (h) or strip width (w).

In the case of cascaded coupled lines increasing phase shift occurs at the expense of bandwidth since the structure resembles a multi-pole filter. Changing the dielectric constant of the ferroelectric film to change insertion phase also modifies the pass band characteristic, resulting in a net bandwidth of $\approx 10 \%$. The bandwidth compression phenomenon was discussed in [1-3] and [6-7]. The impedance matrix of the cascade network can be derived by traditional coupled line theory using the superposition of even and odd mode excitation [10]. Then an equivalent S-parameter model can be extracted and used to predict the pass-band characteristics of the phase shifter.

Line capacitance (C) can be calculated by adapting the quasi-TEM variational expression from Koul and Bhat [11] and using the transverse transmission line method of Crampagne, Ahmadpanah and Guiraud [12]. 



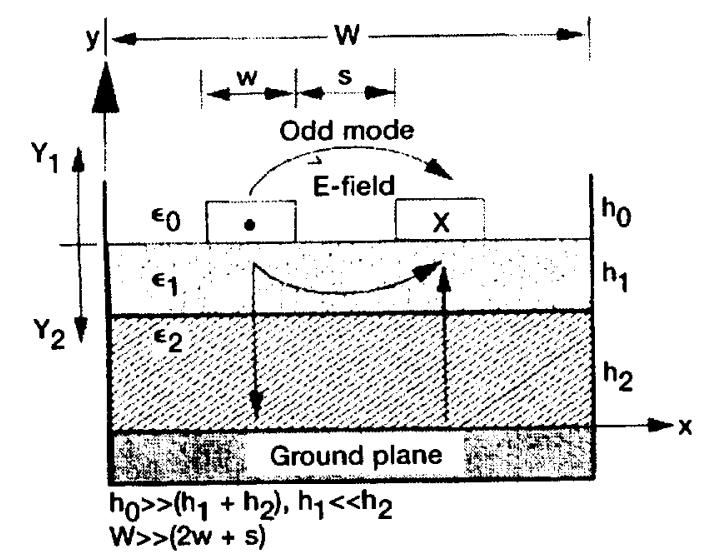

Figure 1.-Cross section of a coupled microstripline phase shifter. The ferroelectric film and substrand thickness are $h_{1}$ and $h_{2}$, respectively. Maximum voltage coupling occurs when the line length $(\ell)$ is $\lambda_{g} / 4$.

$$
\mathrm{l} / \mathrm{C}=\left(\mathrm{l} / \mathrm{q}^{2}\right) \int_{\mathrm{s}}^{\mathrm{s}} \rho(\mathrm{x}, \mathrm{y}) \dot{\phi}(\mathrm{x}, \mathrm{y}) \mathrm{dl}
$$

where $q=\int_{s} \rho(x, y) d l$ and $\phi(x, y)$ is the potential distribution which satisfies Poisson's equation and can be expressed in terms of the admittances $\left(\mathrm{Y}_{1,2}\right)$ at the charge plane $\left(y=h_{1}+h_{2}\right)$ looking in the positive and negative $y$ directions. According to Crampagne, et al., the admittance can be expressed in terms of the dielectric constant of each layer. The charge distribution $\rho(x, y)$ for the even and odd mode excitations was assumed to have the form:

$$
\begin{aligned}
& p(x, y)_{e, o}=1 / w\left\{1+A_{e . o} \mid(2 / w)\left(x-(W-s-w) /\left.2\right|^{3}\right\},\right. \\
& (W-s) / 2-w<x<(W-s) / 2 \\
& Y(n)=\varepsilon_{0} \cdot \operatorname{coth}\left(n \cdot \pi \cdot \frac{h_{0}}{W}\right)+\varepsilon_{0} \cdot \varepsilon_{1} \cdot \frac{\left[\varepsilon_{2} \cdot \varepsilon_{0} \cdot \frac{\left(\operatorname{coth}\left(n \cdot \pi \cdot \frac{h_{3}}{w}\right)+\varepsilon_{2} \cdot \tanh \left(n \cdot \pi \cdot \frac{h_{2}}{w}\right)\right)}{\left(\varepsilon_{2}+\operatorname{coth}\left(n \cdot \pi \cdot \frac{h_{3}}{W}\right) \cdot \tanh \left(n \cdot \pi \cdot \frac{h_{2}}{w}\right)\right)}+\varepsilon_{0} \cdot \varepsilon_{1} \cdot \tanh \left(n \cdot \pi \cdot \frac{h_{1}}{W}\right)\right]}{\left[\varepsilon_{0} \cdot \varepsilon_{1}+\varepsilon_{2} \cdot \varepsilon_{0} \cdot \frac{\left(\operatorname{coth}\left(n \cdot \pi \cdot \frac{h_{3}}{W}\right)+\varepsilon_{2} \cdot \tanh \left(n \cdot \pi \cdot \frac{h_{2}}{W}\right)\right)}{\left(\varepsilon_{2}+\operatorname{coth}\left(n \cdot \pi \cdot \frac{h_{3}}{W}\right) \cdot \tanh \left(n \cdot \pi \cdot \frac{h_{2}}{W}\right)\right)}\right.}
\end{aligned}
$$

where $A_{e, o}$ are constants derived by maximizing the even (e) and odd (o) mode capacitance [11]. That is, a trial function that maximizes capacitance yields the most accurate result. It is an attribute of the variational method that the trial function for the charge distribution does not have to be known precisely a priori in order to evaluate capacitance.

The variational approach is only valid for electrically thin stratified substrates. But since the coupling to surface waves represents an operational limit, it is appropriate for practical microwave applications. The limit was analyzed in [7].

In the even mode currents in the strips are equal in amplitude and flowing in the same direction. In the odd mode currents in the strips are equal in amplitude but flow in opposite directions. So, $Z_{n e}$ is the characteristic impedance of one strip to ground with equal currents in the same direction and $Z_{n o}$ is the characteristic impedance of one strip to ground with equal currents in opposite directions. The microstrip mode exists when $s>h, w$ and $Z_{o c}=Z_{o o}$. The thin FE film is most effective when the phase velocity is dominated by the odd mode fields. The propagation constant is given by $\beta=$ $\omega / v_{p}=\left(\pi / \lambda_{a}\right)\left(\varepsilon_{\text {EVEN }}{ }^{1 / 2}+\varepsilon_{O D D}{ }^{1 / 2}\right)$ where $\varepsilon_{\text {EVEN }}=C_{E} / C_{\text {Eair }}$ and $\varepsilon_{O D D}=C_{O} / C_{O}$ air and $C_{E a i r}$ and $C_{O a i r}$ are obtained by replacing all dielectrics with air (i.e., $\varepsilon_{\mathrm{r}}=1$ ). The even and odd mode dielectric constants are implicitly taken as functions of voltage.

A fairly general formulation for the admittance consisting of an additional dielectric layer, say air, with thickness $h_{3}$ between the host substrate and the ground plane can be where $\varepsilon_{\mathrm{l}}=\varepsilon(\mathrm{V})(1-\mathrm{j} \tan \delta(\mathrm{V}))$

Finally, the odd mode capacitance becomes:

$$
C_{O}=\frac{\left(1+\frac{A_{0}}{4}\right)^{2}}{\sum_{n} g(n) \cdot\left(L(n)+A_{0} \cdot M(n)\right)^{2}}
$$

And the even mode capacitance is similarly obtained, by summing over even integers with $A_{e}$ replacing $A_{0}$. For a TEM transmission line, the characteristic impedance is obtained as $\mathrm{Zo}=\left[(\mathrm{CCair})^{1 / 2} \mathrm{c}\right]^{-1}$ where $\mathrm{c}$ is the speed of light in vacuum.

In general, minimum attenuation is obtained when the effect of the ground plane loss is minimized (i.e., in the odd or balanced mode current flows into one strip and returns through the other). Maximum attenuation occurs in the even or unbalanced mode when equal currents flow into both strips and return through ground.

A comparison between the quasi-TEM approximation and a full-wave electromagnetic simulation is given in Table I for microstrip. The spacing $s$ was allowed to increase just until the even and odd mode capacitance was equivalent. Choosing arbitrarily large values for $\mathrm{s}$ yields anomalous results.

TABLE I. MODELED DATA FOR A $2 \mu \mathrm{m}$ FERROELECTRIC LAYER ON $.25 \mathrm{~mm}$ THICK LaAlO $3, w=75 \mu \mathrm{m}, \mathrm{s} \gg \mathrm{w}, \mathrm{h}$ (MICROSTRIP MODE).

\begin{tabular}{ccc}
\hline $\begin{array}{c}\text { Er Ferroelectric } \\
\text { Laver }\end{array}$ & Eeff (Sonnet $\left.{ }^{\mathrm{TM}}\right)$ & Eeff(Variational) \\
\hline 300 & 18.76 & 18.43 \\
600 & 21.34 & 21.00 \\
900 & 23.49 & 23.09 \\
1200 & 25.41 & 24.93 \\
1500 & 27.18 & 26.59 \\
1800 & 28.84 & 28.12 \\
\hline
\end{tabular}



For coupled strip lines using the superposition of the even and odd modes: $Z_{11}=Z_{22}=-j / 2\left(Z_{O e}+Z_{O n}\right) \cot \theta(V)$ and $Z_{13}=$ $Z_{31}=-j / 2\left(7_{O_{c}}-7_{O_{0}}\right) \csc \theta(V)$ where $\theta(V)$ is the voltage dependent electrical length of the coupled lines (i.e., $\theta(\mathrm{V})=$ $\beta(V) C)$. The characteristic impedance can be expressed as the geometric mean of the even and odd mode impedance such that $Z_{O}=\left(Z_{O e} Z_{O_{n}}\right)^{1 / 2}$. This is strictly valid only for pure TEM propagation and ignores frequency dependence. However, for a practical geometry with moderate coupling, like that considered here, the expression is appropriate. To facilitate the calculation, the $\mathrm{Z}$-parameters are converted into $\mathrm{ABCD}$ or chain parameters for the cascaded sections. The conversion is $A_{f}=Z 11_{f} Z Z 21_{f}, B_{f}$ $=\left(Z 11_{f} \cdot Z 22_{f}-Z 12_{f} \cdot Z 21_{f}\right) / Z 21_{f}, C_{f}=1 / Z 21_{f}, D_{f}=Z 22_{f} / Z 21_{f}$ where the indices are used to show frequency dependence. If the short intervening sections of microstrip line (between each coupled line section) are assumed to be of zero length the overall two-port chain matrix becomes:

$$
\left(\begin{array}{ll}
a_{f} & b_{f} \\
c_{f} & d_{f}
\end{array}\right)=\left(\begin{array}{ll}
A_{f} & B_{f} \\
C_{f} & D_{f}
\end{array}\right)^{N}
$$

where $\mathrm{N}$ is the number of sections. Finally, the two-port $\mathrm{S}$-parameters can be converted back according to:

$$
\begin{gathered}
s 11_{f}=\frac{a_{f}+\frac{b_{f}}{Z_{o}}-\left(c_{f} \cdot Z_{o}\right)-d_{f}}{a_{f}+\frac{b_{f}}{Z_{o}}+c_{f} \cdot Z_{o}+d_{f}} \\
s 21_{f}=\frac{2}{a_{f}+\frac{b_{f}}{Z_{o}}+c_{f} \cdot Z_{o}+d_{f}}
\end{gathered}
$$

Experiments have shown that the insertion phase shift is roughly proportional to $\mathrm{t}^{0.67}$. Tables II and III compare film thickness effects on different substrates and are in excellent agreement with experiment.

TABLE II.-THEORETICAL PROPAGATION CHARACTERISTICS OF A

\begin{tabular}{|c|c|c|c|c|c|c|c|c|c|}
\hline \multirow[t]{2}{*}{$\varepsilon_{1}$} & \multicolumn{3}{|c|}{$\mathrm{h}_{1}=2 \mu \mathrm{m}$} & \multicolumn{3}{|c|}{$h_{1}=1 \mu \mathrm{m}$} & \multicolumn{3}{|c|}{$h_{1}=0.5 \mu \mathrm{m}$} \\
\hline & $\overline{\phi_{1}{ }^{n}}$ & $\begin{array}{c}\alpha d \\
(\mathrm{~Np} / \mathrm{m})\end{array}$ & $\begin{array}{c}\text { Zo } \\
(\Omega)\end{array}$ & $\phi_{1}{ }^{\circ}$ & $\begin{array}{c}\alpha d \\
(\mathrm{~Np} / \mathrm{m})\end{array}$ & $\begin{array}{c}\mathrm{Zo} \\
(\Omega)\end{array}$ & $\phi_{1}{ }^{\circ}$ & $\begin{array}{c}\alpha \mathrm{d} \\
(\mathrm{Np} / \mathrm{m})\end{array}$ & $\begin{array}{c}\text { Zo } \\
(\Omega) \\
\end{array}$ \\
\hline 2500 & 65.9 & 66.3 & 29.7 & 50.5 & 45.3 & 37.9 & 40.0 & 30.7 & 46.7 \\
\hline 1000 & 46.6 & 22.5 & 40.7 & 37. & 15.3 & 49.6 & 31.2 & 10.3 & 58.4 \\
\hline 500 & 37.3 & 3.0 & 49.7 & 31.2 & 2.2 & 58.4 & 27.1 & 1.6 & 66.4 \\
\hline
\end{tabular}
SINGLE COUPLED MICROSTRIP SECTION ON $0.3 \mathrm{~mm} \mathrm{MgO.}$ $l=350 \mu \mathrm{m}, \mathrm{s}=10 \mu \mathrm{m}$, AND $\mathrm{w}=30 \mu \mathrm{m}$.

In all cases the loss tangent of the host substrate was 0.001 and the loss tangent for the ferroelectric film of thickness $h_{1}$ was taken as $0.05,0.028$, and 0.005 for $\varepsilon_{1}$ equal to 2500,1000 , and 500 , respectively. The net phase shift is 2.2 times greater for the $2 \mu \mathrm{m}$ film compared to the $500 \mathrm{~nm}$ film. Table III summarizes propagation characteristics for a coupled microstrip section on $h_{2}=0.25 \mathrm{~mm} \mathrm{LaAlO}(\varepsilon=24)$. The insertion phase is greater because the effective permittivity of the composite structure is substantially greater than that of Table II. But the correlation between phase shift and film thickness is about the same.

The model slightly underestimates total phase shift possibly due to contributions from the microstrip interconnects and anisotropic permittivity (i.e., higher in-plane permittivity). The dielectric loss, characteristic impedance, and passband characteristics are consistent with data extracted from experiments in [1-3] and [6-7].

TABLE III.-THEORETICAL PROPAGATION CHARACTERISTICS

\begin{tabular}{|c|c|c|c|c|c|c|c|c|c|}
\hline \multirow[t]{2}{*}{$\varepsilon_{1}$} & \multicolumn{3}{|c|}{$h_{1}=2 \mu \mathrm{m}$} & \multicolumn{3}{|c|}{$\mathrm{h}_{1}=1 \mu \mathrm{m}$} & \multicolumn{3}{|c|}{$\mathrm{h}_{1}=0.5 \mu \mathrm{m}$} \\
\hline & $\phi_{1}{ }^{\prime \prime}$ & $\begin{array}{c}\alpha d \\
(\mathrm{~Np} / \mathrm{m})\end{array}$ & $\begin{array}{c}\text { Zo } \\
(\Omega)\end{array}$ & $\phi_{1}{ }^{\prime \prime}$ & $\begin{array}{c}\alpha d \\
(\mathrm{~Np} / \mathrm{m})\end{array}$ & $\begin{array}{l}\text { Zo } \\
(\Omega)\end{array}$ & $\phi_{1}{ }^{\prime \prime}$ & $\begin{array}{c}\alpha d \\
(\mathrm{~Np} / \mathrm{m})\end{array}$ & $\begin{array}{c}\text { Zo } \\
(\Omega)\end{array}$ \\
\hline 2500 & 100.1 & 70.4 & 24.4 & 79.4 & 47.9 & 30.1 & 65.3 & 32.3 & 35.9 \\
\hline 1000 & 73.9 & 23.9 & 32.1 & 61.7 & 16.2 & 37.8 & 53.6 & 10.8 & 43.0 \\
\hline 500 & 61.4 & 3.4 & 37.9 & 53.5 & 2.5 & 43.1 & 48.2 & 1.8 & 47.4 \\
\hline
\end{tabular}
OF A SINGLE COUPLED MICROSTRIP SECTION ON $0.25 \mathrm{~mm}$ LaAIO, $l=457 \mu \mathrm{m}, \mathrm{s}=8 \mu \mathrm{m}$, and $w=25 \mu \mathrm{m}$

\section{Conclusions}

A theoretical model for thin film ferroelectric phase shifters has been developed and has been shown to be in good agreement with experiment and electromagnetic simulators. The insertion phase shift and pass-band characteristics can be closely approximated. The significance of ferroelectric film thickness was examined. A key advantage of this technolngy is the relatively large feature size. Whereas the GaAs FET performance is largely dictated by transconductance and hence carrier transit time across the gate region, the coupled line phase shifters are static devices. The electrode gap separation $(\approx 10 \mu \mathrm{m})$ determines the degree of electromagnetic coupling and the dc potential required to tune the film. The loss tangent of a thin dielectric film $\left(h_{1} \leq 2 \mu \mathrm{m}\right)$ on a good substrate (tan $\delta$ $\leq 0.001)$ can deteriorate substantially $(\tan \delta \leq 0.05)$ before the dielectric loss dominates total loss. If the $\tan \delta$ of the ferroelectric film could be maintained at 0.005 or less, the films contribution to total loss would be negligible except for the mismatch it introduces as it is tuned.

\section{REFERENCES}

[1] F. Van Keuls, et al., "A Ku-Band Gold/Ba $\mathrm{Sr}_{1-x} \mathrm{TiO}_{3} / \mathrm{LaAlO}_{3}$ Conductor/Thin Film Ferroelectric Microstrip Line Phase Shifter for Room Temperature Communications Applications," Microwave and Optical Technology Letters, vol. 20, pp. 53-56, Jan. 1999.

[2] F. Van Keuls, et al., "YBar $\mathrm{Cu}_{3} \mathrm{O}_{7}$ \&. Au/ $\mathrm{SrTiO}_{3} / \mathrm{LaAlO}_{3}$ Thin Film Conductor/Ferroelectric Coupled Microstripline Phase Shifters for Phased Array Applications," Appl. Phys. Lett., vol. 71, pp. 3075-3077, Nov. 1997.

[3] R.R. Romanofsky, et al., "A K-Band Linear Phased Array Antenna Based on $\mathrm{Ba}_{(0,6)} \mathrm{Sr}_{(0,4)} \mathrm{TiO}_{3}$ Thin Film Phase Shifters", IEEE MTT Symposium, Boston, MA, June 2000.

[4] J. Mantese, et al., "Applicability of Effective Medium Theory to Ferroelectric/Ferromagnetic Composites With Composition and Frequency Dependent Complex Permittivities and Permeabilities". J. Appl. Phys., vol. 79, pp. 1655-1660, Feb. 1996.

[5] Q. Jia, et al., Appl. Phys. Lett., vol. 74, pp. 1564-1566, Mar. 1999.

[6] R. Romanofsky, et al., "Analysis and Optimization of Thin Film Ferroelectric Phase Shifters," MRS Symp. Proc., Boston, MA, Dec. 1999.

[7] R.R. Romanofsky, PhD Thesis, Cleveland State University, 1999.

[8] H. Phelan, "Spiraphase Reflectarray for Multitarget Radar," Microwave Joumal, vol. 20, July 1977, pp. 67-73.

[9] J. Huang, et al., "A Ka-Band Microstrip Reflectarray With Elements Having Variable Rotation Angles", IEEE Trans. Antennas and Propagation, vol. 46, May 1998, pp. 650-656.

[10] E. Jones, et al., "Coupled Strip Transmission Line Filters and Directional Couplers," IRE Trans., vol. MTT-4, pp. 78-8I, 1956.

[11] S. Koul et al., "Propagation Parameters of Coupled Microstrip-Line Transmission Lines for Millimeter Wave Applications", IEEE MTT-S Digest, pp. 489-491, 1981.

[12] R. Crampagne, et al., "A Simple Method for Determining the Green's Function for a Large Class of MIC Lines Having Multilayered Dielectric Structures”, IEEE Trans. MTT, vol. 26, pp. 82-87. 1978. 


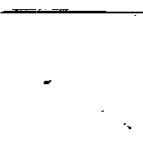

